\title{
A CLASS OF BOUNDED STARLIKE FUNCTIONS
}

\author{
HERB SILVERMAN
}

Department of Mathematics

University of Charleston

Charleston, SC 29424

(Received April 8, 1993)

\begin{abstract}
We consider functions $f(z)=z+\cdots$ that are analytic in the unit disk and satisfy there the inequality $\operatorname{Re}\left(f^{\prime}(z)+z f^{\prime \prime}(z)\right)>\alpha, \alpha<1$. We find extreme points and then determine sharp lower bounds on $\operatorname{Re} f^{\prime}(z)$ and $\operatorname{Re}(f(z) / z)$. Sharp results for the sequence of partial sums are also found.
\end{abstract}

KEY WORDS AND PHRASES. Univalent, starlike.

1991 AMS SUBJECT CLASSIFICATION CODES. Primary 30C45; Secondary 30C50.

\section{INTRODUCTION.}

Denote by $A$ the family of functions $f(z)=z+\cdots$ that are analytic in the unit disk $\Delta=\{z:|z|<1\}$ and by $S$ the subfamily of functions that are univalent in $\Delta$. Let $R$ be the functions $f$ in $A$ for which $\operatorname{Re}\left(f^{\prime}(z)+z f^{\prime \prime}(z)\right)>0, z \in \Delta$. Chichra [1] showed that $R \subset S$. In fact, he proved that $\operatorname{Re} f^{\prime}(z)>0, z \in \Delta$, and hence $R \subset C$, the class of close-to-convex functions. $\mathrm{R}$. Singh and S. Singh [4] showed that $R \subset S^{*}$, the family of starlike functions. They later found in [5] for $f \in R$ and $z \in \Delta$ that $\operatorname{Re}(f(z) / z)>1 / 2$ and that the partial sums $S_{n}(z, f)$ satisfy $\operatorname{Re}\left(S_{n}(z, f) / z\right)>1 / 3$. Neither of these results is sharp.

In this note, we find the sharp bounds. Our results will be put into a slightly more general context. Denote by $R(\alpha), \alpha<1$, the subfamily of $A$ consisting of functions $f$ for which $\operatorname{Re}\left(f^{\prime}(z)+z f^{\prime \prime}(z)\right)>\alpha, z \in \Delta$. Denote by $P(\alpha), \alpha<1$, the subfamily of $A$ consisting of functions $f$ for which $\operatorname{Re} f^{\prime}(z)>\alpha, z \in \Delta$. It was shown in [5] that $R(\alpha) \subset S^{*}$ for $\alpha . \geq-1 / 4$. We improve this lower bound and also find the smallest $\alpha$ for which $R(\alpha) \subset S$. Our approach in this note will be to characterize the extreme points of $R(\alpha)$, which lead to sharp bounds for certain linear problems.

2. MAIN RESULTS.

THEOREM 1. (i) The extreme points of $R(\alpha)$ are

$$
f_{x}(z)=\int_{0}^{z} \frac{(2 \alpha-1) t+(2 \alpha-2) \bar{x} \log (1-x t)}{t} d t,|x|=1 .
$$

(ii) A function $f$ is in $R(\alpha)$ if and only if $f$ can be expressed as

$$
F(z)=\int_{X} f_{x}(z) d \mu(x)
$$

where $\mu$ varies over the probability measures defined on the unit circle $X$.

PROOF of (i). Hallenbeck [2] showed that the extreme points of $P(\alpha)$ are

$$
\{(2 \alpha-1) z+(2 \alpha-2) \bar{x} \log (1-x z),|x|=1\} .
$$


Since $\left(z f^{\prime}\right)^{\prime}=f^{\prime}+z f^{\prime \prime}$, we have $f \in R(\alpha)$ if and only if $z f^{\prime} \in P(\alpha)$. Hence the operator $L$ defined by $L(f)=\int_{0}^{z}(f(t) / t) d t$ is a linear homeomorphism $L: P(\alpha) \rightarrow R(\alpha)$ and thus preserves extreme points.

PROOF of (ii). The family $R(\alpha)$ is convex and is therefore equal to its convex hull. This enables us to characterize $f \in R(\alpha)$ by $F(z)=\int_{X} f_{x}(z) d \mu(x)$.

COROLLARY 1. If $f(z)=z+\sum_{n=2}^{\infty} a_{n} z^{n} \in R(\alpha)$, then $\left|a_{n}\right| \leq 2(1-\alpha) / n^{2}$.

The result is sharp.

PROOF. The coefficient bounds are maximized at an extreme point. Now $f_{x}(z)$ may be expressed as

and the result follows.

$$
f_{x}(z)=z+2(1-\alpha) \sum_{n=2}^{\infty} \frac{x^{n-1} z^{n}}{n^{2}},|x|=1
$$

COROLLARY 2. If $f \in R(\alpha)$, then $|f(z)| \leq(1-\alpha)\left(\frac{\pi^{2}}{3}-1\right)+\alpha$. get

PROOF. From (2.2), we see that $|f(z)| \leq r+2(1-\alpha) \sum_{n=2}^{\infty} \frac{r^{n}}{n^{2}},|z|=r$. Letting $r \rightarrow 1$, we

$$
|f(z)| \leq 1+2(1-\alpha)\left(\frac{\pi^{2}}{6}-1\right)=(1-\alpha)\left(\frac{\pi^{2}}{3}-1\right)+\alpha .
$$

Corollary 2 shows that the family $R(\alpha)$ is bounded in $\Delta$ for all real $\alpha, \alpha<1$, even though its functions may not be univalent. Note from (2.1) that the extreme points of $P(\alpha)$ are unbounded in $\Delta$ for all $\alpha<1$.

In the next two theorems, we will be looking at continuous linear operators $L(f)=R e f^{\prime}$ and $L(f)=\operatorname{Re}(f(z) / z)$ acting on $R(\alpha)$. It therefore suffices to investigate the extreme points in determining minima. Since $R(\alpha)$ is rotationally invariant, we may restrict our attention to the extreme point

$$
g(z)=(2 \alpha-1) z-2(1-\alpha) \int_{0}^{z} \frac{\log (1-t)}{t} d t=z+2(1-\alpha) \sum_{n=2}^{\infty} \frac{z^{n}}{n^{2}}
$$

THEOREM 2. If $f \in R(\alpha)$, then

$$
\operatorname{Re} f^{\prime}(z)>(1-\alpha)(2 \log 2-1)+\alpha \quad(z \in \Delta) .
$$

The result is sharp.

PROOF. We need only consider $g(z)$ defined by (2.3). We have

$$
g^{\prime}(z)=(2 \alpha-1)-2(1-\alpha) \frac{\log (1-z)}{z} \text {. }
$$

In [2] it is shown that

$$
R e-\frac{\log (1-z)}{z} \geq \frac{\log (1+r)}{r},|z|=r
$$

so that $\operatorname{Re} g^{\prime}(z) \geq(2 \alpha-1)+2(1-\alpha) \frac{\log (1+r)}{r}$. Letting $r \rightarrow 1$, the result follows.

The case $\alpha=0$ is found in [5].

COROLLARY 1. $R(\alpha) \subset S$ for $\alpha \geq-\frac{1}{2}\left(\frac{2 \log 2-1}{1-\log 2}\right)=\alpha_{0} \approx-0.63$ and $R(\alpha) \not \subset S$ for $\alpha<\alpha_{0}$.

PROOF. We know that $P(0) \subset S$. Since $(1-\alpha)(2 \log 2-1)+\alpha=0$ for $\alpha=\alpha_{0}$, the first part is a consequence of Theorem 2. The result cannot be extended to $\alpha<\alpha_{0}$ because $g^{\prime}(-1)=0$ at $\alpha=\alpha_{0}$. Thus $g^{\prime}(-r)=0$ for some $r=r(\alpha)<1$ when $\alpha<\alpha_{0}$.

COROLLARY 2. $\sum_{k=1}^{\infty} \frac{\cos k \theta}{k+1} \geq \sum_{k=1}^{\infty} \frac{(-1)^{k}}{k+1}=\log 2-1$. 
PROOF. From (2.3) we have

$$
\operatorname{Re} g^{\prime}(z)=1+2(1-\alpha) \sum_{k=1}^{\infty} \frac{r^{k} \cos k \theta}{k+1},|z|=r
$$

which according to (2.4) and (2.5) is minimized when $\theta=\pi$. We then let $r \rightarrow 1$.

In [5] it is shown that $\operatorname{Re}(f(z) / z)>1 / 2$ for all $f$ in $R$. The next theorem improves this lower bound to $\frac{\pi^{2}}{6}-1 \approx 0.645$. But first we state

LEMMA 1. $\sum_{n=1}^{\infty} \frac{(-1)^{n+1}}{n^{2}}=\frac{\pi^{2}}{12}$.

PROOF. $\sum_{n=1}^{\infty} \frac{1}{n^{2}}-\sum_{n=1}^{\infty} \frac{(-1)^{n+1}}{n^{2}}=2 \sum_{n=1}^{\infty} \frac{1}{(2 n)^{2}}$, so that

$$
\sum_{n=1}^{\infty} \frac{(-1)^{n+1}}{n^{2}}=\frac{1}{2} \sum_{n=1}^{\infty} \frac{1}{n^{2}}=\frac{\pi^{2}}{12}
$$

THEOREM 3. If $f \in R(\alpha)$, then

$$
\operatorname{Re} \frac{f(z)}{z}>(1-\alpha)\left(\frac{\pi^{2}}{6}-1\right)+\alpha \quad(z \in \Delta) .
$$

The result is sharp, with the extremal function $g$ defined by (2.2).

PROOF. Again, we need only consider

$$
\frac{g(z)}{z}=(2 \alpha-1)-2(1-\alpha) \int_{0}^{z} \frac{\log (1-t)}{t z} d t .
$$

Setting $t=v z$, we may write

$$
\frac{g(z)}{z}=(2 \alpha-1)-2(1-\alpha) \int_{0}^{1} \frac{\log (1-v z)}{v z} d v .
$$

Since $\operatorname{Re}\left(-\frac{\log (1-w)}{w}\right) \geq \frac{\log (1+|w|)}{|w|},|w|<1$, we get from (2.6) that for $|z|=r$,

$$
R e \frac{g(z)}{z} \geq(2 \alpha-1)+2(1-\alpha) \int_{0}^{1} \frac{\log (1+v r)}{v r} d v=\frac{g(-r)}{-r} .
$$

But from (2.3) we see that

$$
\frac{g(-r)}{-r}=1+2(1-\alpha) \sum_{n=2}^{\infty} \frac{(-r)^{n+1}}{n^{2}}>1+2(1-\alpha) \sum_{n=1}^{\infty} \frac{(-1)^{n}}{(n+1)^{2}}
$$

An application of Lemma 1 yields

$$
R e \frac{f(z)}{z} \geq \frac{g(-r)}{-r}>1+2(1-\alpha)\left(\frac{\pi^{2}}{12}-1\right)=(1-\alpha)\left(\frac{\pi^{2}}{6}-1\right)+\alpha .
$$

In [5], R. Singh and S. Singh showed that $R(\alpha) \subset S^{*}$ for

$$
\alpha \geq-\frac{1}{2}\left[\inf _{f \in R(\alpha), z \in \Delta} \operatorname{Re}\left(\frac{f(z)}{z}\right)\right]
$$

This enabled them to conclude that $R(\alpha) \subset S^{*}$ for $\alpha \geq-1 / 4$. Our sharp bound in Theorem 3 gives the following improvement.

COROLLARY. $R(\alpha) \subset S^{*}$ for $\alpha \geq \frac{6-\pi^{2}}{24-\pi^{2}} \approx-0.2738$.

PROOF. The result follows from Theorem 3 upon solving the inequality

$$
\alpha \geq-\frac{1}{2}\left((1-\alpha)\left(\frac{\pi^{2}}{6}-1\right)+\alpha\right)
$$


The next lemma, due to Rogosinski and Szegö, will be needed for our results on partial sums.

LEMMA 2 [3]. $\sum_{k=1}^{n} \frac{\cos k \theta}{k+1} \geq-\frac{1}{2}$.

THEOREM 4. Denote by $S_{n}(z, f)$ the $n$th partial sum of a function $f$ in $R(\alpha)$. If $f \in R(\alpha)$, then

(i) $S_{n}(z, f) \in P(\alpha)$,

(ii) $\operatorname{Re} \frac{S_{n}(z, f)}{z}>\frac{1+\alpha}{2}, \quad z \in \Delta$.

The results are sharp, with extremal function $g(z)$ defined by (2.3) and $n=2$.

PROOF of (i). As before, it suffices to prove our results when $f(z)=g(z)$. We have

$$
S_{n}^{\prime}(z, g)=1+2(1-\alpha) \sum_{k=2}^{n} \frac{z^{k-1}}{k}=1+2(1-\alpha) \sum_{k=1}^{n-1} \frac{r^{k} \cos k \theta}{k+1} .
$$

By Lemma 2 and the minimum principle for harmonic functions,

$$
\operatorname{Re} S_{n}^{\prime}(z, g)>1+2(1-\alpha)\left(-\frac{1}{2}\right)=\alpha
$$

PROOF of (ii). We have

$$
\operatorname{Re} \frac{S_{n}(z, g)}{z}=1+2(1-\alpha) \sum_{k=1}^{n-1} \frac{r^{k} \cos k \theta}{(k+1)^{2}} .
$$

Since $1 /(k+1)$ is decreasing, we use Lemma 2 and summation by parts to obtain

$$
\sum_{k=1}^{n-1}\left(\frac{1}{k+1}\right)\left(\frac{\cos k \theta}{k+1}\right) \geq \frac{1}{2}\left(-\frac{1}{2}\right)=-\frac{1}{4} .
$$

Substituting inequality (2.8) into (2.7) and applying the minimum principle, we get

$$
\operatorname{Re} \frac{S_{n}(z, g)}{z}>1+2(1-\alpha)\left(-\frac{1}{4}\right)=\frac{1+\alpha}{2} .
$$

In the special case $\alpha=0$, (i) gives the result found in [5] and (ii) improves the estimate of $1 / 3$ to the sharp bound of $1 / 2$.

REMARK. This work was completed while the author was a Visiting Scholar at the University of Michigan.

\section{REFERENCES}

1. CHICHRA, P.N., New subclasses of the class of close-to-convex functions, Proc. Amer. Math. Soc. 62 (1) (1977), 37-43.

2. HALLENBECK, D.J., Convex hulls and extreme points of some families of univalent functions, Trans. Amer. Math. Soc. 192 (1974), 285-292.

3. ROGOSINSKI, W. \& SZEGÖ, G., Uber die Abschimlte Vol potenzreihen die in ernein Kreise be schrarikt bleiben, Math. Z. 28 (1928), 73-94.

4. SINGH, R. \& SINGH, S., Starlikeness and convexity of certain integrals, Ann. Univ. Mariae Curie-Sklodowska Sect A 35 (1981), 45-47.

5. SINGH, R. \& SINGH, S., Convolution properties of a class of starlike functions, Proc. Amer. Math. Soc. 106 (1989), 145-152. 


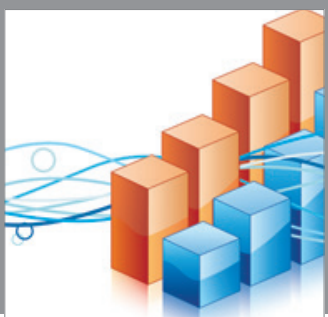

Advances in

Operations Research

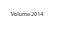

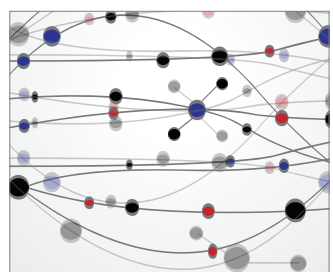

\section{The Scientific} World Journal
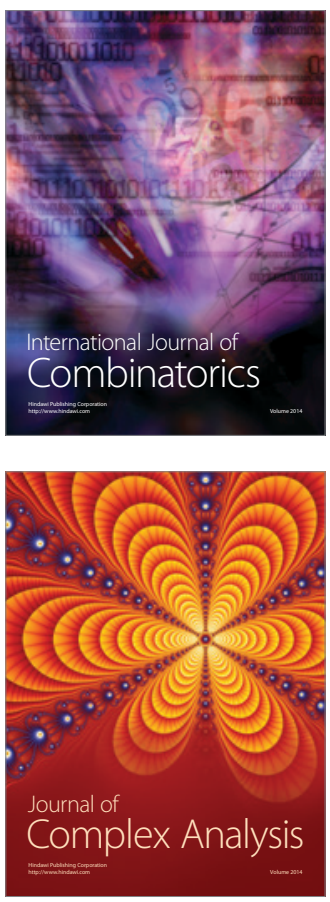

International Journal of

Mathematics and

Mathematical

Sciences
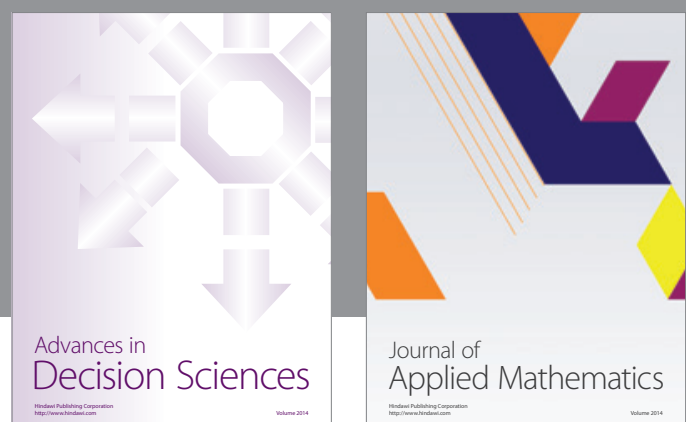

Journal of

Applied Mathematics
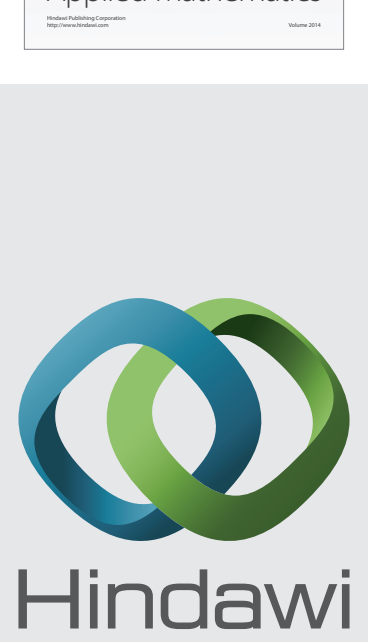

Submit your manuscripts at http://www.hindawi.com
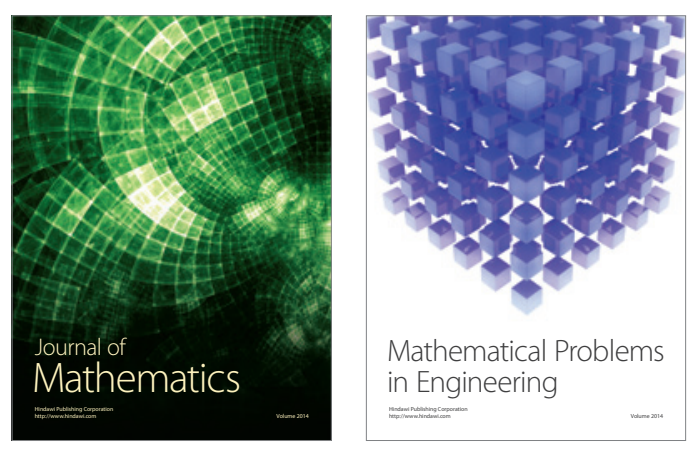

Mathematical Problems in Engineering
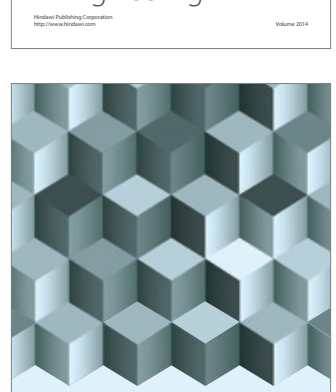

Journal of

Function Spaces
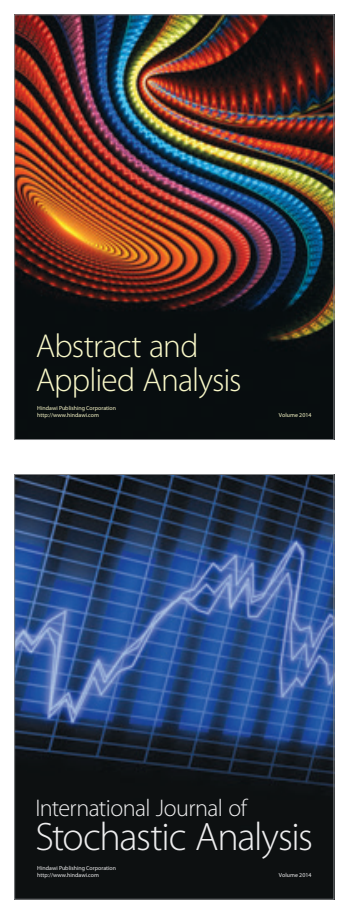

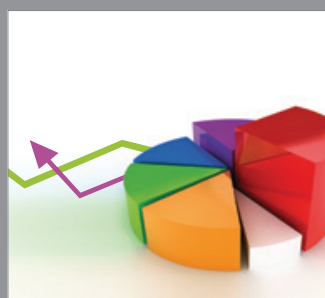

ournal of

Probability and Statistics

Promensencen
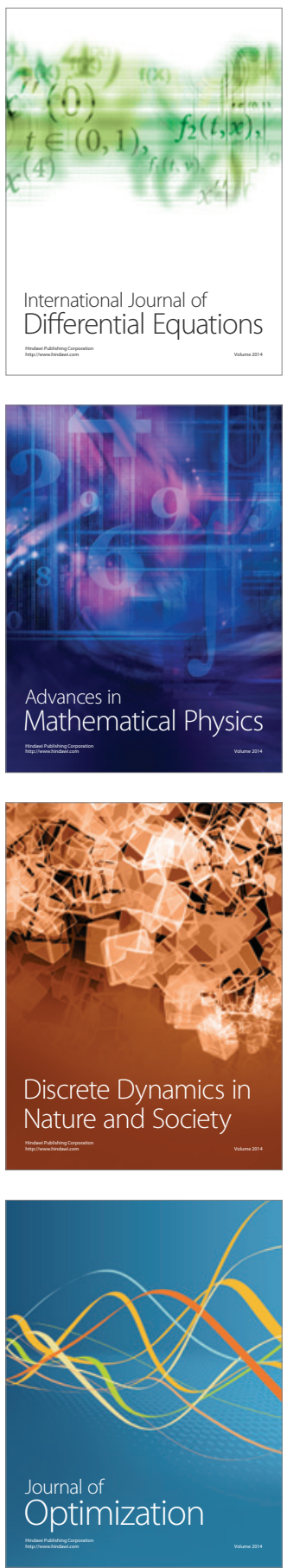